\title{
Damage function for historic paper. Part II: Wear and tear
}

\author{
Matija Strlič ${ }^{\text {* }}$, Carlota M. Grossi ${ }^{1}$, Catherine Dillon ${ }^{1}$, Nancy Bell ${ }^{2}$, Kalliopi Fouseki ${ }^{1}$, Peter Brimblecombe ${ }^{3}$, \\ Eva Menart ${ }^{1}$, Kostas Ntanos ${ }^{2}$, William Lindsay ${ }^{2}$, David Thickett ${ }^{4}$, Fenella France ${ }^{5}$ and Gerrit De Bruin ${ }^{6}$
}

\begin{abstract}
Background: As a result of use of library and archival documents, defined as reading with handling in the context of general access, mechanical degradation (wear and tear) accumulates. In contrast to chemical degradation of paper, the accumulation of wear and tear is less well studied. Previous work explored the threshold of mechanical degradation at which a paper document is no longer considered to be fit for the purpose of use by a reader, while in this paper we explore the rate of accumulation of such damage in the context of object handling.

Results: The degree of polymerisation (DP) of historic paper of European origin from mid-19th-mid-20th Century was shown to affect the rate of accumulation of wear and tear. While at DP $>800$, this accumulation no longer depends on the number of handlings (the process is random), a wear-out function could be developed for documents with DP between 300 and 800. For objects with DP <300, one large missing piece (i.e. such that contains text) developed on average with each instance of handling, which is why we propose this DP value as a threshold value for safe handling.

Conclusions: The developed model of accumulation of large missing pieces per number of handlings of a document depending on DP, enables us to calculate the time required for an object to become unfit for use by readers in the context of general access. In the context of the average frequency of document use at The UK National Archives (Kew), this period is 60 years for the category of papers with DP 300, and 450 years for papers with DP 500. At higher DP values, this period of time increases beyond the long-term planning horizon of 500 years, leading to the conclusion that for such papers, accumulation of wear and tear is not a significant collection management concern.
\end{abstract}

Keywords: Heritage management, Conservation, Fitness for use, Wear and tear, Mechanical properties, Reliability engineering

\section{Background}

In continuous incremental degradation processes, such as degradation by chemical reactions whose rate is influenced by heat and humidity, or due to the effect of light/ UV or pollution, material change is most often modelled deterministically. Examples of such modelling are doseresponse functions, and the most recent review for historic paper was published in 2011 [1]. These presume that by knowing previous states of the material and all model parameters, material properties can be uniquely

\footnotetext{
*Correspondence: m.strlic@ucl.ac.uk

${ }^{1}$ Institute for Sustainable Heritage, University College London, London, UK

Full list of author information is available at the end of the article
}

determined at any point in time. All objects that are exactly the same thus degrade in precisely the same way under the same conditions. This is a reasonable assumption that can be used in modelling, provided that we know the most important physical properties of historic objects that affect their degradation.

Physical degradation, i.e. degradation leading to mechanical failure as a consequence of excessive or repetitive mechanical stress e.g. due to handling, is rarely studied in collections. A notable exception is environmentally-induced mechanical stress, particularly in relation to objects made of wood and painted objects, where humidity fluctuations lead to internal stresses that result in cracking and other forms of mechanical failure [2,3].
Chemistry Central (c) 2015 Strlič et al. This article is distributed under the terms of the Creative Commons Attribution 4.0 International License (http://creativecommons.org/licenses/by/4.0/), which permits unrestricted use, distribution, and reproduction in any medium, provided you give appropriate credit to the original author(s) and the source, provide a link to the Creative Commons license, and indicate if changes were made. The Creative Commons Public Domain Dedication waiver (http://creativecommons.org/ publicdomain/zero/1.0/) applies to the data made available in this article, unless otherwise stated. 
The probability of mechanical failure increases as a result of constant physical and chemical degradation of materials as they become weaker and therefore more susceptible to damage by fatigue and fracture. Incremental degradation of materials does not result in a sudden change, but it does increase the probability of failure due to fatigue. The reliability engineering concept described as the so-called 'bathtub curve' [4,5] conceptualises these phenomena showing the rate of failure in a population of objects as a function of time and consisting of three phases. Of interest here will be the second and the third phase, when wear and tear accumulates either at a constant rate, or possibly at increased rates as the flexibility of cellulosic fibres decreases with loss of their degree of polymerisation (DP). It is useful to stress that mechanical properties of certain paper types, such as coated or heavily sized, may significantly depend on additives, however, such paper types are not of interest to this research.

For paper-based material, brittleness is often discussed as the most important consequence of environmentally induced chemical degradation. A number of collection surveys performed since 1970 s onwards, have relied on a fairly straightforward, although destructive, manual folding test [6]. In this test, a page corner is folded as many times it takes for it to break off on gentle pulling.

The 1988 Swedish study [7] was one of the most thorough early studies, and used sixfolds as the criterion to categorise paper into the most degraded, with "many tears and/or pieces missing, worn edges, slight to strong yellowing, breaking corner on sixfolds". Using these criteria, it was estimated that the proportion of books with paper in the worst state was approximately the same in the National Archives of Sweden and in the Uppsala University Library, i.e. $20 \%$. These were mainly from the period 1860-1890, which coincides with the introduction of mechanised rosin sizing across much of Europe in 1850s [8]. In similar studies at the Library of Congress, $30 \%$ of collections were estimated to be no longer fit for the purpose of manual handling, and similarly, $40 \%$ in the Harvard University Library and $26 \%$ in the Stanford University Library [9]. Other recent studies [10] continue to report similar values.

The premise of such studies is not based on an understanding of what users might perceive as an unfit document [11], but rather on the assessment of the current 'conservation state' of the document and, in some cases, of the risk of damage by handling, as perceived by conservation experts. Such perceived risk, however, still needs to be quantitatively justified by a study showing how wear and tear accumulates during use. Additionally, some of the above mentioned surveys are based on assessment elements, which users clearly do not perceive as unacceptable change, e.g. yellowing or discolouration, tears or minor missing pieces [11].
There have been many attempts to correlate mechanical properties of paper with a more easily measurable quantity. It has been estimated that with a degree of polymerisation of 250, paper becomes too brittle even to be tested for its tensile strength [12]. In a recent study in collaboration between the National Archives of the Netherlands, Sweden and Slovakia and the Royal Library of the Netherlands [13], an attempt was made to quantify the folding test results and sixfolds were found to correspond to a DP of approximately $\sim 350$. The uncertainty associated with the manual test was found to be significant, so interpretation of the result requires some caution.

Further related values have recently been reported. In a study of iron gall ink drawings, conservators assessed that the risk of drawings becoming damaged during handling by non-expert users was too high at DP $\sim 400$ [14]. In a study of cellulose-based painting canvases, involving a panel of 19 conservation experts, it was estimated that the risk of painting transportation and handling became too high if the DP $<400$ [15].

However, neither the minimum number of folds nor the corresponding DP value, as determined by conservation experts, reflect how wear and tear is accumulated during handling of books and archival objects, i.e. the 'material wear-out' phase of the bathtub curve [4].

Such a wear-out function for library and archival materials would need to be able to predict the point of loss of fitness for use for general access, i.e. in a reading room or an exhibition (e.g. reading with handling, or display), discussed in the first paper in this series [11]. It was shown that neither discolouration nor tears matter to users, while deterioration considered unacceptable for the purpose of reading (with handling) or display is related to missing pieces of text. It is therefore necessary to study how missing pieces accumulate during the use of paper with different mechanical properties.

Previous research has demonstrated a correlation between DP and mechanical properties such as tensile strength and fold endurance [16]. While highly brittle paper documents continue to retain value as 'museum objects' even after their life as an object of general utility has been spent [17], it is important to stress that our interest is in library and archival use in general access conditions, i.e. without supervision.

Paper of very low DP has low fold endurance and tensile strength and is experienced by readers as brittle and difficult to handle. Therefore, it could be assumed that there is an association between DP and the occurrence of physical changes associated with mechanical properties over a given number of challenges (e.g. tears, loose sheets, missing pieces and folds which result in breaks). It could also be assumed that there is an association between DP and the extent of use required before such 
changes become classed as damage. Such a function, combined with the frequency of use in an experimental case study, may reveal that regardless of its brittleness, a document could continue to be fit for a considerable length of time if the frequency of its use (reading, display) is low. In other words, if a document is not used at all, its brittleness is not a concern. This paper thus addresses the accumulation of wear and tear.

\section{Methods}

\section{Experimental design}

For the purpose of the case study, a set of mock documents (bound, i.e. books, and unbound, i.e. loose sheets) made from historic paper of known chemical and mechanical properties were subjected to physical challenges representative of handling that might be typical of a historic archive or library collection. After rounds of physical challenges (ten instances of handling each), the documents were assessed for changes to their physical state, i.e. deterioration. Of particular interest were tears and missing pieces, as their influence on user evaluation of fitness for use was assessed in our previous research [11].

The experimental design was expected to yield the following types of data:

1. The number or degree of changes per challenge, i.e. ten instances of handling (e.g. X number of large or small tears, Y number of large or small missing pieces).

2. Given that the challenges were intended to be representative of handling in a real library or archival setting, and the frequency of use in archives/libraries is generally known, then the above data can be converted to expected number of changes per period of time.

3. The above data and analysis was obtained for bound and unbound documents and was used to explore the comparison between a typical library document and a typical archival folder.

The SurveNIR Reference Historic Paper Collection [18] was used as the source of paper. The textbooks, guidebooks and fiction books range from mid-nineteenth to mid-twentieth century, and are written in Slovenian, Croatian, Serbian, German and Italian and 25 were selected for the simulation study on the basis of the DP of the paper in the books ( 300-1000) and the amount of paper available, as both a bound and an unbound object had to be produced using the same paper. The paper was characterised in terms of variables such as molecular weight, DP, $\mathrm{pH}$, fibre furnish and other properties, using traditional analytical techniques, such as viscometry for
DP and cold extraction for $\mathrm{pH}$ [19]. Since some of the papers used in this study contained lignin and could not be dissolved in the solvent used for viscometry, their DP was determined using size exclusion chromatography by Morana RTD d.o.o. (Ivancna Gorica), following the previously described methodology [20]. The average uncertainty of DP determination was $\sim 10 \%$, which included experimental and sampling uncertainties and results of this research need to be interpreted in light of this uncertainty.

It also needs to be clarified that all papers used in this study were made following the European papermaking practices of the time (rosin sizing), acidic and not coated. All were printed.

From among the total of 25 pairs of bound/unbound simulated objects, 4 were additionally degraded in $\mathrm{HCl}$ enriched atmosphere at $80{ }^{\circ} \mathrm{C}$ overnight, in order to rapidly obtain highly brittle material. The DP of these papers was measured using the same technique as outlined in [19], following ISO 5351:2010. As this research is only concerned with the material state (measured in terms of DP) at the time of the experiment described in "Challenges", previous environmental history (or indeed ultra-accelerated degradation in $\mathrm{HCl}$-atmosphere) of the experimental papers is not of interest.

From each SurveNIR book, a $0.5-0.7 \mathrm{~cm}$ stack of paper (several sections) was rebound by a professional bookbinder (sections hand-sewn, spine glued with PVAc, hard binding on board, including an end paper, mull spine lining, fabric cover over spine, uncovered, with overhang, paper trimmed around the edges). This type of binding enabled all books to be comparable and data independent of binding type.

Most of the books had 6 sections, giving 48 sheets or 96 pages per document. For one book there was only enough paper to rebind 2 sections, giving 16 sheets or 32 pages. For two bound documents there was only enough paper to rebind 3 sections, giving 24 sheets or 48 pages.

For each of the 25 bound documents, there was a matching document made of unbound paper from the same SurveNIR sample book, with sections cut along the inner margin to create sets of loose sheets, which were additionally trimmed around the borders. Using guidance on storage of loose-sheet documents provided by The UK National Archives, Kew (TNA), the unbound documents have been placed in four flap folders. The unbound documents and folders have been labelled with their original SurveNIR code and the pages have been renumbered. Figure 1 shows some examples.

Eight sets of three pairs (bound and unbound, same paper) of documents were compiled, with one pair of documents set aside as replacement if needed. In each set of three pairs there was a range of paper DP values (low, 

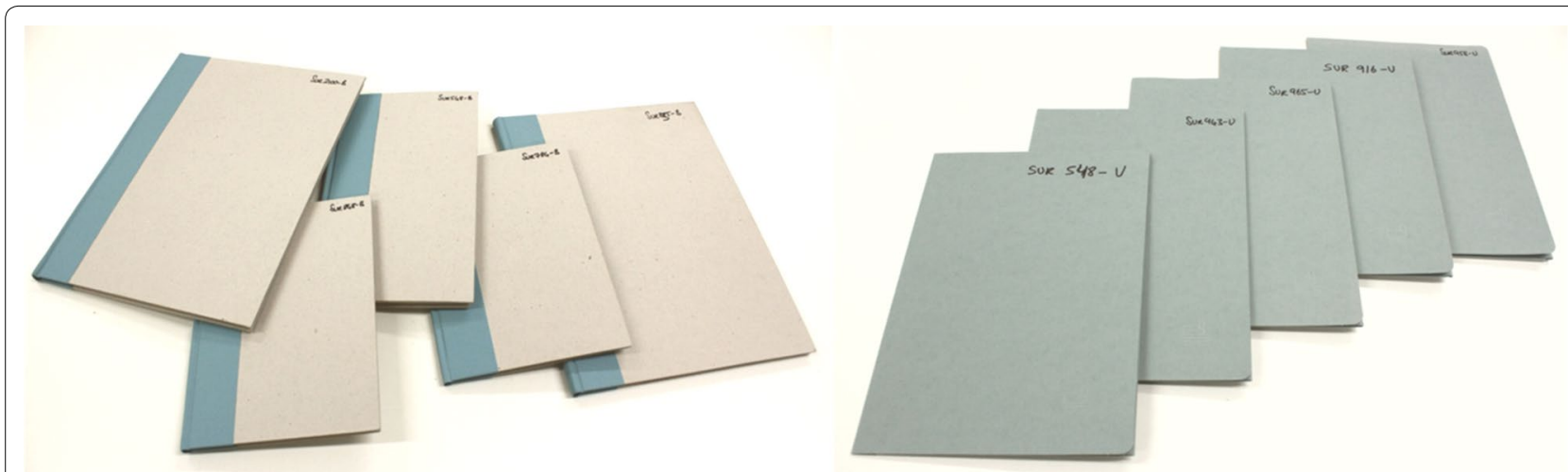

Fig. 1 Examples of bound sample books and unbound paper in folders, as used in the study

medium and high DP pairs) in order to avoid confounds of DP with any physical challenges.

Each set of documents was packaged in a large envelope that prevented wear and tear during transportation.

\section{Challenges}

The documents were subjected to blocks of physical challenges. Each challenge had the following components:

- Packing.

- Tasks performed by participants, involving transport to the challenge venue (office or home), unpacking, performing a task with each document, repacking and transport back to the lab.

- Assessment of each document by the researcherassessor.

For a block of physical tasks (i.e. a challenge) the aim was to subject each document to 10 instances of handling. A number of challenge cycles was conducted, mostly 9, representing 90 instances of handling. Each challenge on a given document was performed by a different participant. Their brief was to leaf through the document 10 times as if reading/searching for information. Some paper was too brittle and allowed for only 5 challenges before assessments of change became very onerous due the extent of physical degradation (Fig. 2).

To simulate reading with handling, participants were asked to leaf through an entire document in a short space of time. It took about 1-2 h to complete the tasks for a set of documents. The 9 participants were members of staff, researchers and volunteers (non-experts) at UCL Institute for Sustainable Heritage.

To make the study as representative as possible, the participants were assigned to challenges using a random number generator. Additionally, in each challenge, a participant received three books and three archival folders with paper of low, medium and high DP. No participant was assigned the same object twice.

Wear and tear could also occur during transportation of documents within an institution. However, the characteristics of transport and handling occurring in real historic

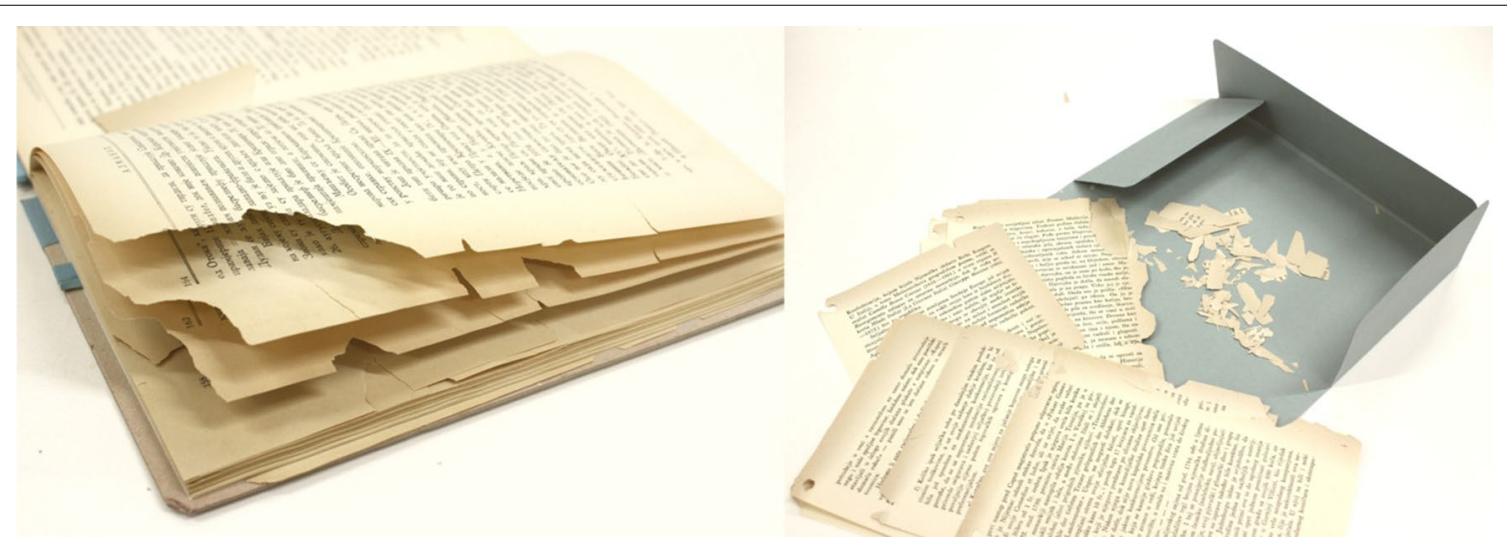

Fig. 2 The consequences of five challenges (50 instances of handling representing reading with handling) for the most brittle paper 
libraries and archives vary from institution to institution, and potentially within an institution. For example, an archival folder might be retrieved from the repository, used very briefly by a reader, and then returned to the repository on the same day. Another box might be retrieved and used intensively for a week before being returned to the repository. In addition, different parts of a collection are held in different locations and produced to different reading rooms. Because of this, potential mechanical deterioration between the challenges was avoided by packing the documents into sturdy envelopes.

\section{Assessment}

Assessment took place at the end of every block of physical challenges. The key measured features followed the elements of distress as discussed in Part I [11]: tears and missing pieces. These were classified according to size and location:

- Small: tears of less than $5 \mathrm{~mm}$ from the edge of a sheet.

- Medium: tears or missing pieces in the 5-20-mm boundary, or tears that affect the inner margin in a book (binding edge).

- Large: tears or missing pieces that cross over the 20-mm boundary, within which text/images would be affected.

It is important to note that the assessment method used in this study is not 'condition' assessment [5] but rather a quantitative measure of physical change, specifically designed to enable categorisation in terms of the fitness of a document.

Since tears and missing pieces are caused by handling, their occurrence should be correlated with the mechanical properties of paper. Therefore the assessment measures are intended to be both indicators of use and sensitive to paper mechanical strength.

To avoid assessor bias, the assessment methods were developed and piloted in collaboration with several assessors and researchers initially. A number of books were independently assessed by two assessors on two occasions. To standardise the assessments, the following tools were developed:

- Templates. These were necessary as the sheets were trimmed to remove any previous wear and tear, which was assumed to have an effect on the experiment. Two assessment templates were designed: a template for bound documents and a template for unbound documents, printed onto a transparency and placed over the sheet being assessed.

- The template divides a sheet into several regions: $\mathrm{A}-\mathrm{H}$ (Bound) and A-I (Unbound). These are over- laid on a 1-cm grid used for measuring the length of tears and the area of missing pieces. The red-dotted line marks the 5-mm boundary around the edge of a sheet (within which features are only counted). The solid red line represents a 2 -cm margin (Fig. 3).

- The template represents a typical sheet from a book based on measurements taken before rebinding (e.g. most bottom margins were $>2 \mathrm{~cm}$ wide), but could be moved around in order to assess sheets of differing dimensions.

- A template excel file was created to record the data and each spreadsheet had the following elements:

- Sheet: features were recorded sheet by sheet.

- Tears: tears around the edge of the sheet within the 5 -mm boundary were counted (likely to be approximate); other tears were recorded individually (location, length, direction etc.). Since small tears can develop into large tears and large tears can lead to missing pieces and, therefore, the elimination of tears, changes in tear numbers could be positive as well as negative.

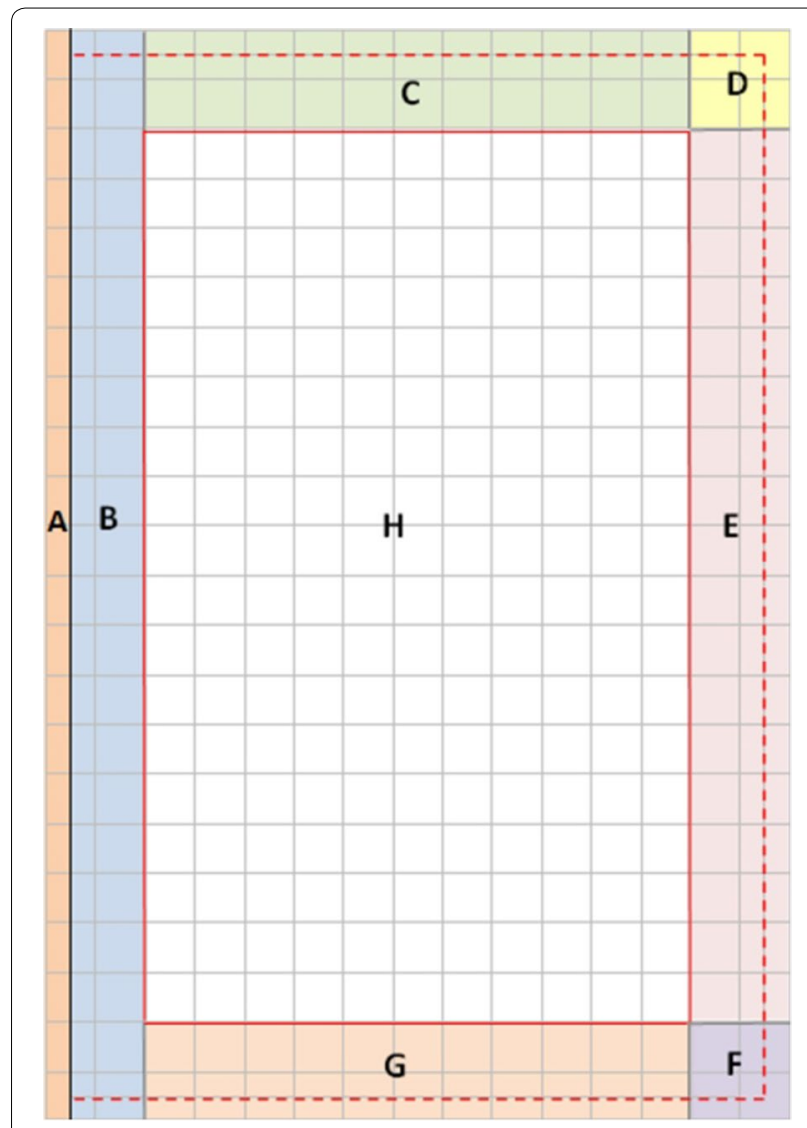

Fig. 3 The template used in the assessment of wear and tear for bound volumes boundary $A$ represents the inner (binding) margin 
- Missing pieces: similarly to tears, small missing pieces were counted and larger ones were described in detail, categorised and summed up.

Based on piloting, there was greater agreement between assessors about the location of a feature and whether the text area was affected, rather than finer details of features (e.g. whether a tear has affected the text and images in a document, rather than how long and in what direction the tear is). These are also likely to be the features most important to readers when assessing fitness for use [11]. Hence, these were the key measures used to describe change in the documents over time.

Following the pilot assessment phase, all changes (tears, missing pieces) in all document sets were assessed by the same researcher after each challenge.

Frequency of document use at the National Archives, Kew Data on the frequency of use were made available by The National Archives, Kew, per bay (set of shelves) and not down to any greater detail, e.g. shelf, box or reference number. The data illustrate that yearly productions of individual documents would be expected to be very low, with $50 \%$ of the c. 20,000 bays at Kew having documents produced from them between 1 and 10 times per year (Fig. 4).

\section{Results and discussion}

\section{Overall deterioration frequency analysis}

Nine participants were involved in the experiments, carrying out a challenge per week. The introduced

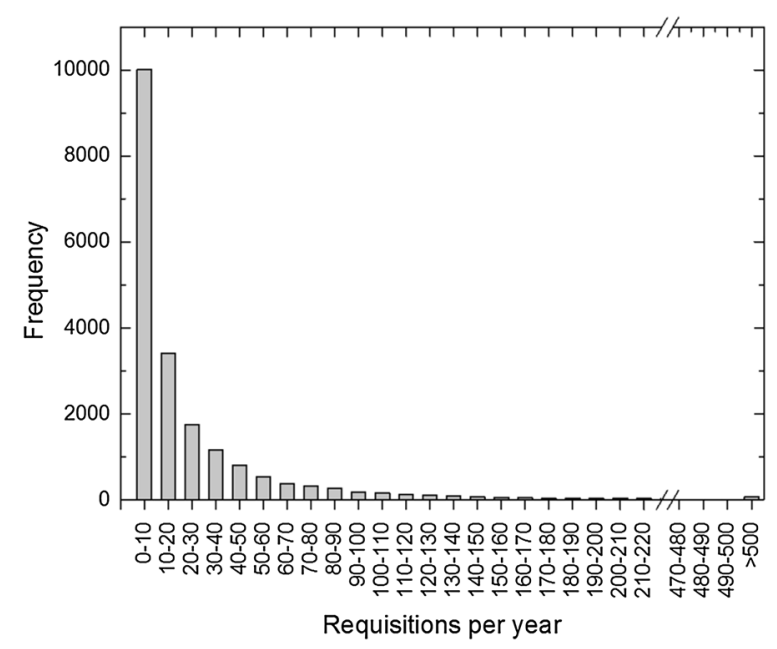

Fig. 4 Frequency plot representing the number of uses (requisitions) per bay, per year, at The National Archives, Kew. Documents from 10,019 bays are requested $0-10$ times per year, and from 77 bays $>500$ times per year wear and tear was comparable across the nine participants, as demonstrated in Fig. 5. The plot represents average fractions (percentages) of deterioration in an item they 'treated' compared to all observed deterioration in the same item, caused by all of the individual participants.

It is of interest to note that in some cases the introduced deterioration was negative, which is reasonable since in the calculation of total deterioration, medium and large tears and missing pieces were added up. Since a medium tear may transform into a large tear or a large tear into a missing piece, different aspects of wear and tear may accumulate or transform into other aspects of deterioration and thus disappear. Small tears were not considered in the calculation as in our previous study [11] it was shown that it is predominantly large elements of degradation that lead to loss of fitness.

Since not all participants treated all the documents, and some may have treated the more fragile ones and others not (the most fragile four documents were only treated 50 times, all others 90 times), bias could have been introduced in the data. However, the data in Fig. 5 shows that it is difficult to systematically discriminate between the participants. While P1 introduced most deterioration on average, it is quite clear that the scatter of data is high. While P6 on average introduced less deterioration, second only to the most careful P7, the maximum deterioration they caused was the second most intensive.

\section{Accumulation of mechanical deterioration}

Interestingly, wear and tear accumulates approximately linearly with the number of challenges, as shown in Figs. 6 and 7. This again indicates that there is probably insignificant difference between the participants and the force they applied to objects. More importantly, this allows us to describe the data and develop functions showing the accumulation of aspects of deterioration as a function of the number of challenges (and by interpolation, the number of times an object was handled, resembling requisitions in an archival or a library context). The linear relationship between aspects of deterioration and the number of challenges also indicates that accumulation of wear and tear can be treated as an incremental process.

It is of interest to note that tears develop at a similar rate in bound and unbound items, while missing pieces develop at a much lower rate for bound items than unbound ones. Although this observation indicates that for books and archival items different dose-response functions could be developed, all the observations were used in the following calculations (Fig. 8) due to data scatter. It is also possible that further differences could be found for object of different size, weight or type of 

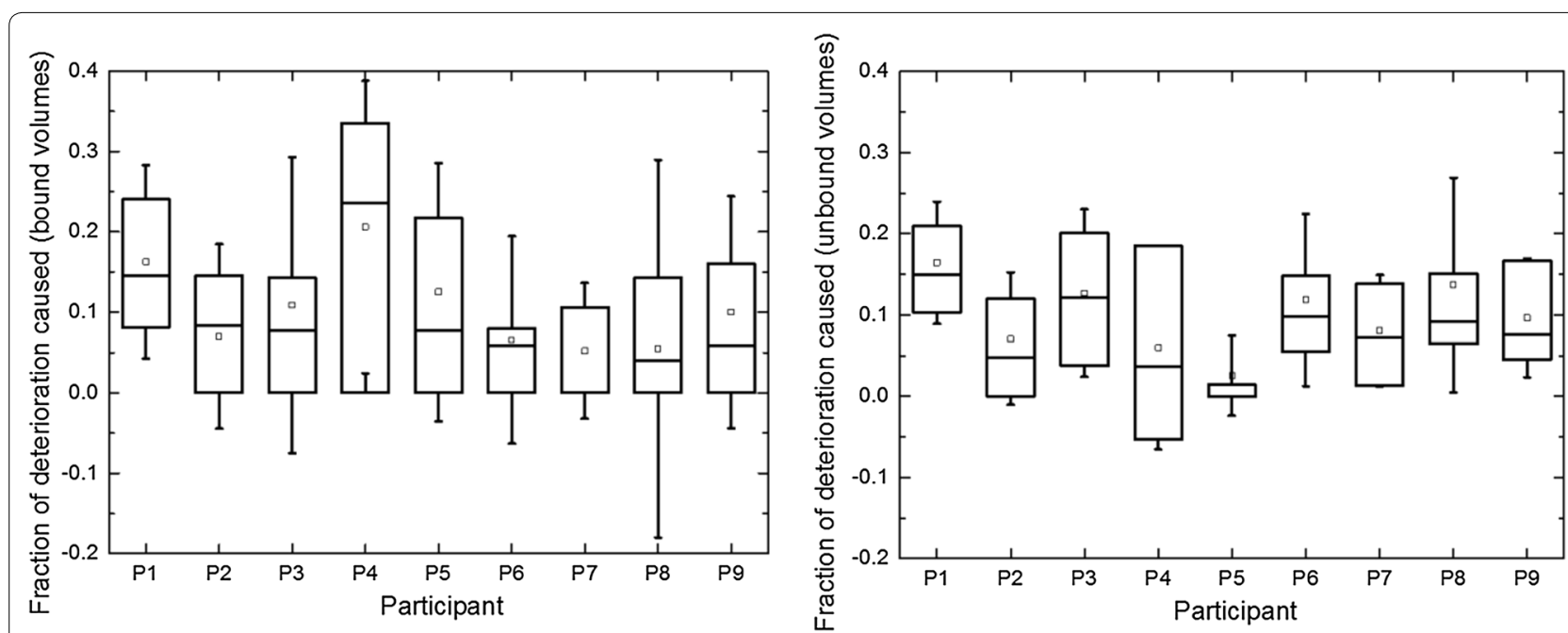

Fig. 5 Boxplots comparing the fractions of the total number of elements of deterioration (small and large, tears and missing pieces) per document, caused by individual participants (P1-P9). Left bound (books); right unbound documents (archival folders). A boxplot shows the means (open square), the standard deviation (whiskers), whereas the boxes represent the first and the third quartile and the median. Left bound documents; right unbound documents
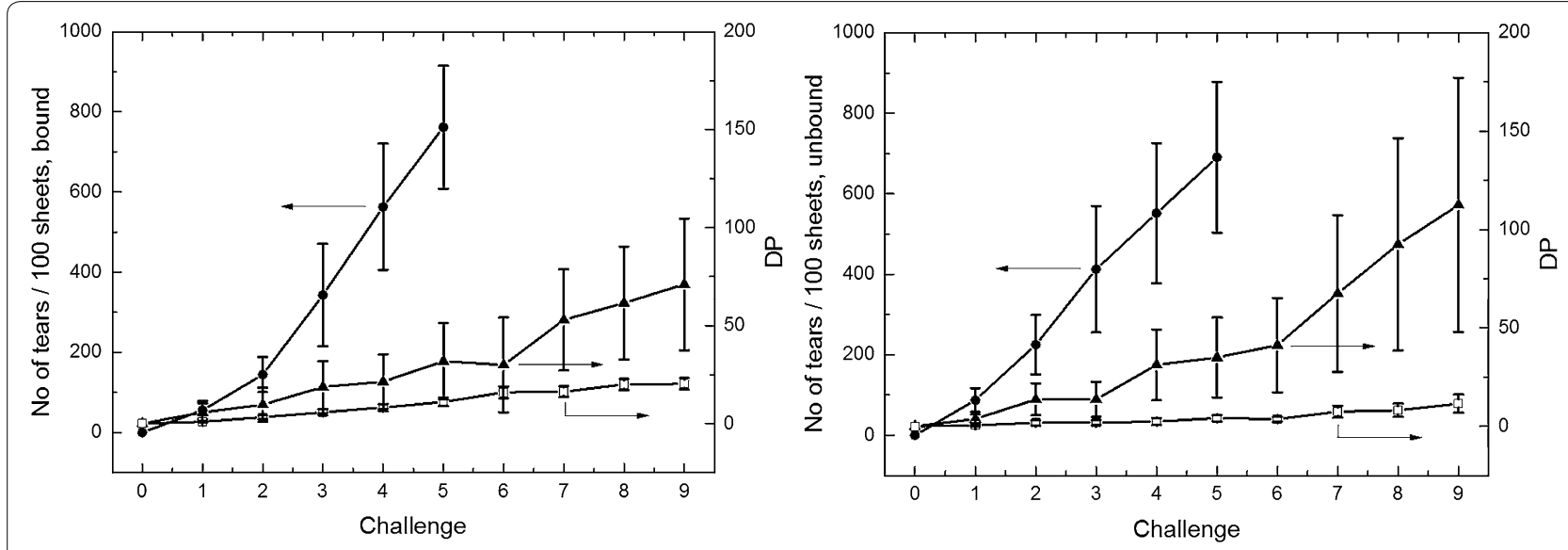

Fig. 6 Accumulation of tears (all sizes) calculated per 100 sheets during the 5-9 challenges (50-90 treatments/instances of handling). Left bound volumes, right unbound folders. The left $y$-axis shows values for the DP interval: triangle $-\mathrm{DP}<300(\mathrm{~N}=5)$, the right $y$-axis for all other DP intervals: circle $-300<\mathrm{DP}<600(\mathrm{~N}=5)$, open square $-600<\mathrm{DP}<900(\mathrm{~N}=7)$. For DP $>900$, no mechanical deterioration was caused. The error bars represent the standard deviation for the average number of tears observed in the individual DP interval

binding; however, this was outside the scope of this research.

The finding that missing pieces accumulate more slowly than tears is also useful, and it might be reasonable to assume that missing pieces develop as a function of tears. This turns out to be the case and in Fig. 8, the number of large missing pieces accumulated during a challenge is plotted as a function of the number of tears in the preceding challenge, but only with two DP categories: $\mathrm{DP}<300$ and $\mathrm{DP}>300$, as the number of missing pieces accumulating in paper with DP $>300$ is very small in comparison.
This is an interesting finding, as according to the threshold fitness for use, discussed in our previous paper [11] it is a large missing piece which makes a document unfit (unless a user has attached a strong historic value to the document, in which case such deterioration is generally viewed with more tolerance).

Extending this definition to a collection of sheets in a library or archival item (with potentially several hundred sheets) is possible by assuming that all textual information in the item is of equal importance.

In practical terms and on average, a large missing piece does not occur in isolation. In a document of 100 sheets, the 

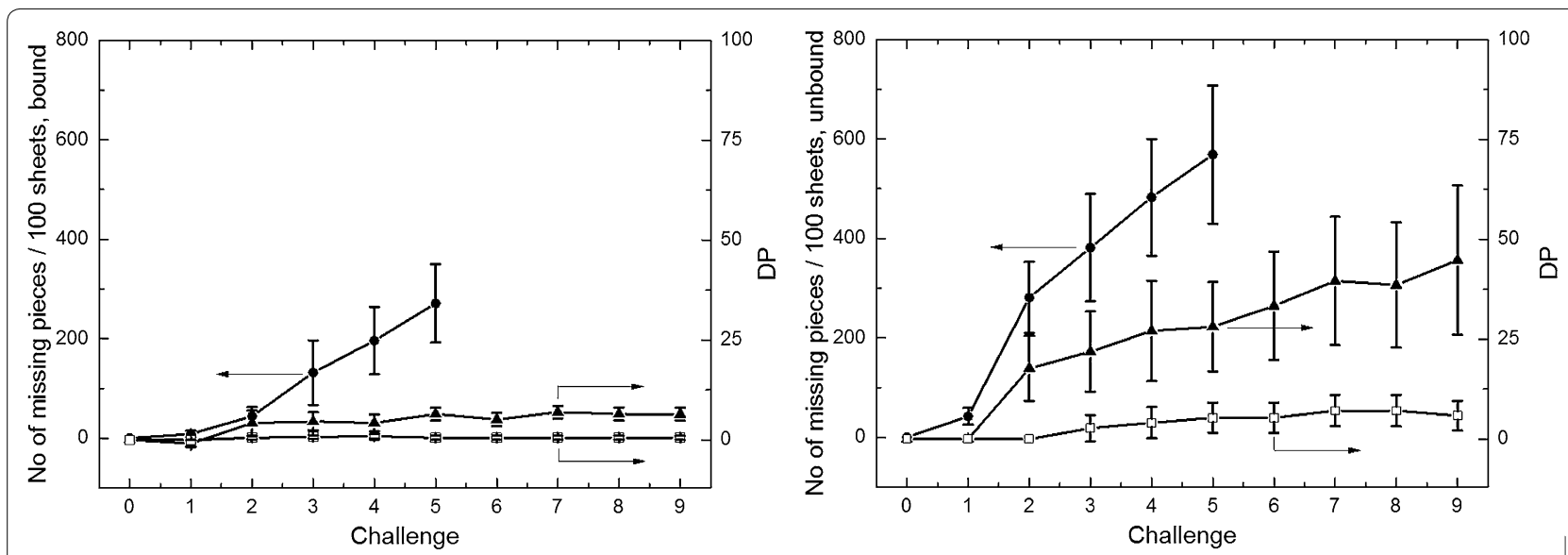

Fig. 7 Accumulation of missing pieces (all sizes) calculated per 100 sheets during the up to 9 challenges ( 90 treatments). Left bound volumes, right unbound folders. The left y-axis shows values for the DP interval: triangle - DP <300, the right y-axis for all other DP intervals: circle $300<\mathrm{DP}<600$, open square $-600<\mathrm{DP}<900$. For DP $>900$, no mechanical deterioration was caused. The error bars represent the standard deviation for the average number of missing pieces observed in the individual DP interval

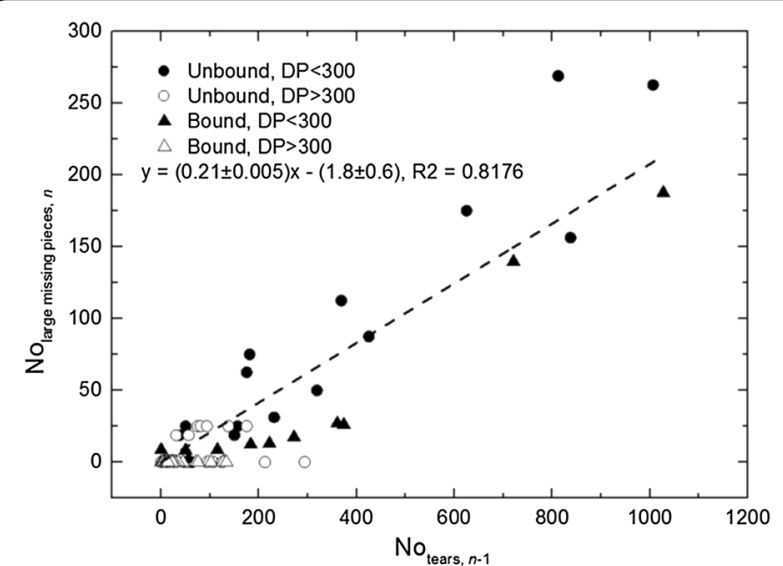

Fig. 8 Accumulation of large missing pieces in the challenge number $n$, as a function of the number of all tears in the preceding challenge ( $n-1)$, both per 100 sheets, for bound and unbound volumes. The regression line is drawn using all data points

first large piece will go missing after approximately 5 tears accumulate in a given sheet, which is already quite extensive wear and tear, not unlike the examples depicted in Fig. 2.

Appreciable accumulation of missing pieces (defined here as one per handling or more, per 100 sheets, where 'handling' refers to one 'instance of handling' as used in this study, i.e., leafing through the entire document once as if reading/searching for information) only occurs in objects with DP < 300: only $2-5 \%$ of all missing pieces accumulate in paper with DP $>300$, all the rest (95-98 \%) in paper with DP $<300$ (Fig. 7). The value of DP 300 could therefore serve as a useful threshold value of DP for the estimation of risk of accumulation of missing pieces and reaching the end of fitness for reading/handling, which is in agreement with the literature discussed in the introduction.

\section{Wear-out function}

Based on the collected data, we can now develop functions looking at both how large missing pieces develop as a function of the DP of paper as well as with the number of handlings or time. The latter depends on the frequency of production in an archive and for this, we need data on the frequency of production, as discussed in "Frequency of document use at the National Archives, Kew". The wear-out function represents the final period of the bathtub curve $[4,5]$ leading to loss of fitness.

In a real environment it may take many years before objects begin to lose large missing pieces, if the frequency of production is low, and especially if DP $>300$. Therefore, to assess the period of time that might be needed to lose a large missing piece, both the frequency of production and the DP of paper will play a role.

Even objects with very low DP will not deteriorate mechanically (i.e. develop tears and missing pieces) if they are not handled-the period of time until loss of fitness for use in the context of general access in a reading room will therefore be extremely long (if not 'indefinite'). However, it is worth stressing that the process of wear and tear accumulation and the process of chemical deterioration (DP loss) are here treated as independent, which works in most cases: if objects are already very deteriorated (DP < 300) then further DP loss will not increase the rate of wear and tear accumulation, and if the objects are not very deteriorated (DP > 500) then the rate of accumulation is insignificantly low in comparison. It is only for objects that are highly accessed and nearing 

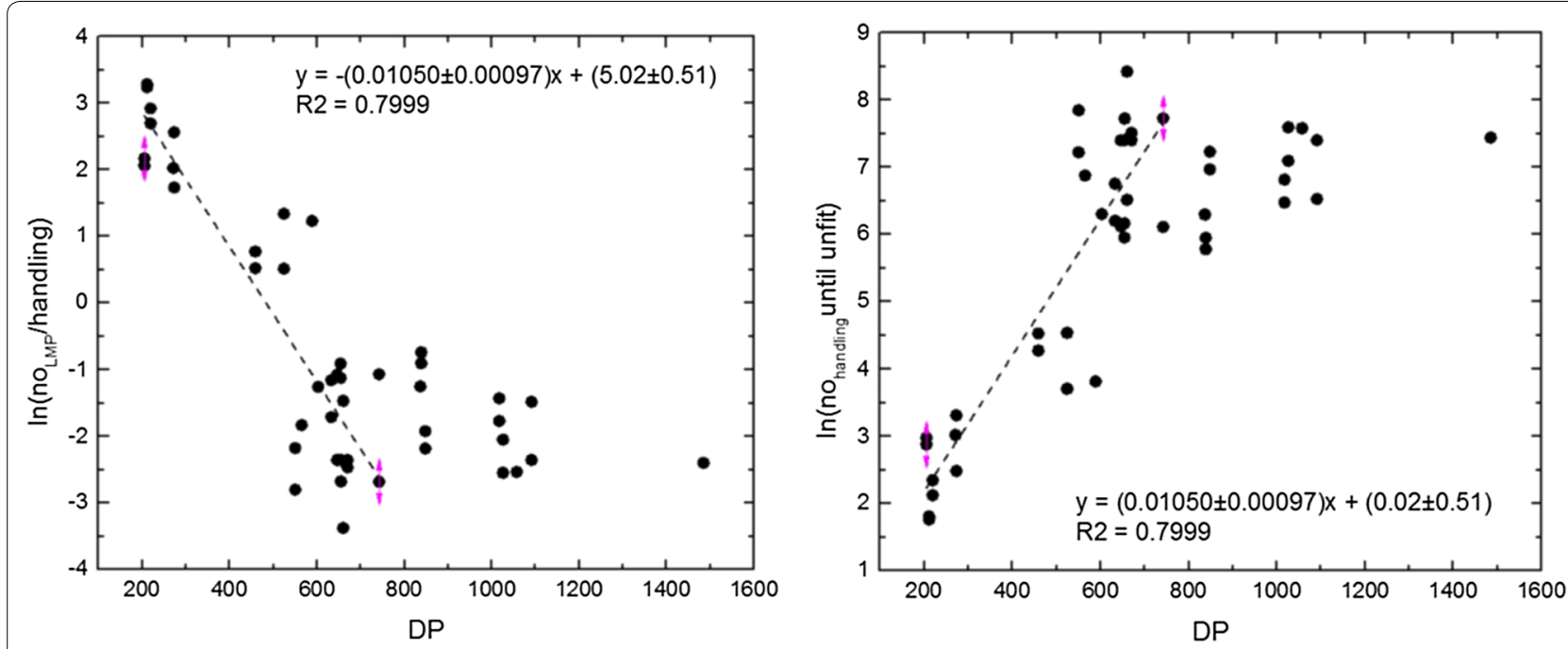

Fig. 9 Left accumulation of large missing pieces (text missing) per handling, i.e. leafing through the entire document once as if reading/searching for information, of paper (per 100 sheets) depending on the DP of paper. Right number of handlings until such paper develops one large missing piece per 100 sheets, depending on their DP. LMP large missing pieces (i.e. a piece with text missing)

the threshold of DP 300 that the two functions would need to be used in conjunction.

In Fig. 9 (left), we show data on the rate of documents becoming unfit, i.e. developing at least one large missing piece per 100 sheets, as they are handled (leafing through the entire document once as if reading/searching for information). The cases where objects did not develop a missing piece, were not taken into account in this calculation (i.e. all objects with DP > 800). The data for bound and unbound objects are combined in these calculations, and a logarithmic function has been used as a model due to the large observed differences.

Figure 9 (right) shows the number of handlings until a document becomes unfit for use, depending on the DP of paper. In order to characterize the state of 'unfit for use' a threshold number of missing pieces per 100 sheets needs to be defined. This is a collection management decision, and in this calculation, one large missing piece per 100 sheets was taken as the threshold of fitness.

The equations describing functions in Fig. 9 are:

$$
\begin{array}{r}
\ln \left(\text { no }_{L M P} / \text { handling }\right)= \\
\cdot(0.01050 \pm 0.00097) \\
\cdot \mathrm{DP}+(5.02 \pm 0.51)
\end{array}
$$

and

$$
\begin{aligned}
& \ln \left(\text { no }_{\text {handling }} \text { until unfit }\right)=(0.01050 \pm 0.00097) \\
& \quad \cdot \mathrm{DP}+\ln \left(\text { threshold }_{L M P} / 100 \text { sheets }\right)-(0.02 \pm 0.51)
\end{aligned}
$$

where DP is $200-800$. For DP $>800$, the value no longer increases, indicating that the process of accumulation of wear and tear no longer depends on DP. In reliability engineering terms, this would relate to the 'random failure phase'.

With this last equation, it is now possible to calculate the average time required for a document with a given DP to deteriorate to the point where it accumulates one large missing piece per 100 sheets (Table 1).

It is evident that for documents with DP $>500$, wear and tear is generally not an important issue, as the period of time until loss of fitness is comparable or longer to the planning horizon $[11,21]$. However, for objects with DP $<500$, wear and tear increases with importance as the DP decreases. It is important to note that even low-DP objects still survive a lot of handling, and that given how infrequently most documents are handled, there is very little loss of fitness even for documents that are considered fragile (DP < 300).

\section{Conclusions}

Wear and tear, as a form of physical degradation, accumulates with use, and ultimately leads to objects becoming unfit for use. The principles described by reliability engineering (bathtub curve) were used to describe the process of accumulation of wear and tear, as a function of time. For the typical type of paper representative of $\sim 70-80 \%$ of Western library and archival collections, we looked at the 'random failure phase' when material weakens, as well as at the 'wear-out failure phase' during which reduction in DP becomes so pronounced that wear and tear (mechanical failure) becomes increasingly likely. If the frequency of object use is known, the curve can be experimentally determined. 
Table 1 Period of time until documents with a given DP become unfit for use assuming average frequency of use at The National Archives (Kew), i.e. $\mathbf{0 . 4 2 5}$ times per document per year, and with the threshold of one large missing piece per 100 sheets

\begin{tabular}{lc}
\hline DP & Years to unfit \\
\hline 300 & 55 \\
400 & 160 \\
500 & 450 \\
600 & 1300 \\
700 & 3700 \\
800 & 10,000 \\
\hline
\end{tabular}

In an experiment involving handling of mock archival and library items, we explored how wear and tear accumulates during handling challenges resembling reading. We conclude the following:

- The accumulation of wear and tear depends on DP for paper with DP < 800. For paper with higher DP, mechanical deterioration appears to accumulate at a very low rate, randomly and independently of DP. In our experiments, only $2-5 \%$ of wear and tear accumulated on paper with DP $>300$.

- For paper with DP $<300$, at least one large missing piece accumulates per 100 sheets during each handling. This is accompanied by tearing and other, minor elements of mechanical deterioration. This value of DP could therefore be used as a safe threshold value for handling, if large missing pieces (containing text) are to be avoided. This is in agreement with previous folding test studies.

- Knowing the average frequency of use, it is possible to estimate the period of time a document with a given DP will remain fit for use if regularly handled. At the National Archives (Kew), wear and tear is not seen as an important issue for documents with DP $>600$, where this period of time (400 years in average) is comparable to the long-term planning horizon (500 years).

From a collection management point of view, this research quantitatively confirms the important contribution of wear and tear to the loss of fitness for purpose, for documents of low DP. Taking the frequency of production into account, as well as the threshold number of large missing pieces per 100 sheets, the wear-out function could add several decades to the lifetime of even the most fragile objects (i.e. with DP $<300$ ), while for objects with DP $>500$, wear and tear on paper no longer represents a significant concern. Objects that are not handled do not reach the threshold fitness for use.
The research focused on mid-19th-mid-20th Century paper of Western origin: lignin containing, rosin sized, acidic, printed and non-coated. While our results show some variation in the accumulation of wear and tear for archival folders and bound objects, which in this research we treated as insignificant, it is possible that objects of significantly different size, weight or type of binding would behave substantially different.

The research quantitatively demonstrates how the desired lifetime of an object or a collection can be increased by adjusting the frequency of access, as mechanical deterioration is proportional to the number instances of user access. In the last paper in our series we will look at how DP decreases with time in relation to environmental parameters during storage (doseresponse function) and to see how this could be visualised in ways that might be meaningful to collection managers and researchers alike.

\section{Authors' contributions}

MS, CMG, CD, NB, KF, PB, EM, KN, WL, DT, GdB jointly developed the concept of this work. CMG and CD organised the experiment and developed guidance for assessment. CD, MS and CMG produced the samples. CMG, MS and CD analysed the data. All co-authors contributed to the manuscript. All authors read and approved the final manuscript.

\section{Author details}

${ }^{1}$ Institute for Sustainable Heritage, University College London, London, UK.

${ }^{2}$ The National Archives, Kew, Richmond, Surrey, UK. ${ }^{3}$ University of East Anglia, Norwich, UK. ${ }^{4}$ English Heritage, London, UK. ${ }^{5}$ Library of Congress, Washington, DC, USA. ${ }^{6}$ Nationaal Archief, The Hague, The Netherlands.

\section{Acknowledgements}

The authors are grateful to colleagues who volunteered for the experiment: Tiphaine Bardon, Katherine Curran, Josep Grau, Andrej Kos, Julia Martinez, Keith Razey (in addition to some of the co-authors). We are also grateful to David Stevens of City Binders (London, UK) for his helpful advice.

\section{Competing interests}

The authors declare that they have no competing interests.

Received: 31 July 2015 Accepted: 11 November 2015

Published online: 26 November 2015

\section{References}

1. Menart E, De Bruin G, Strlič M. Dose-response functions for historic paper. Polym Degrad Stab. 2011;96:2029-39.

2. Rachwał B, Bratasz $Ł$, Łukomski M, Kozłowski R. Response of wood supports in panel paintings subjected to changing climate conditions. Strain. 2012;48:366-74

3. Rachwał B, Bratasz Ł, Krzemień L, Łukomski M, Kozłowski R. Fatigue damage of the gesso layer in panel paintings subjected to changing climate conditions. Strain. 2012;48:474-81.

4. Klutke GA, Kiessler PC, Wortman MA. A critical look at the bathtub curve IEEE Trans Reliab. 2003;52:125-9.

5. Strlič M, Thickett D, Taylor J, Cassar M. Damage functions in heritage science. Stud Conserv. 2013;58:80-7.

6. Walker G, Greenfield J, Fox J, Simonoff JS. The Yale Survey: a large-scale study of book deterioration in the Yale University Library. Coll Res Lib. 1985;46:111-32.

7. Palm J, Cullhed P. Deteriorating Paper in Sweden, FoU-projektet för papperkonservering, Report No. 3. Stockholm: 1988. 
8. Hunter D. Papermaking: history and technique of an ancient craft. New York: Dover Publ; 1987.

9. Buchanan S, Coleman S. Deterioration survey on the Stanford university libraries green library stack collection. Coll Res Lib. 1987:48:102-47.

10. Sobucki W, Srewniewska-Idziak B. Survey of the preservation status of the 19 and 20th century collections at the National Library in Warsaw. Restaurator. 2003;24:189-201.

11. Strlič M, Grossi-Sampedro C, Dillon C, Bell N, Fouseki K, Brimblecombe P, Menart E, Ntanos K, Lindsay W, Thickett D, France F, De Bruin G. Damage function for historic paper. Part I: fitness for use. Herit Sci. 2015;3:33.

12. Shroff DH, Stannett AW. A review of paper aging in power transformers. IEEE Proc. 1985;132:312-8.

13. Kolar J, Strlič M. Enhancing the lifespan of paper-based collections, AIC 35th annual meeting. Richmond: 2007.

14. Strlič M, Cséfalvayová L, Kolar J, Menart E, Kosek J, Barry C, Higgitt C, Cassar M. Non-destructive characterisation of iron gall ink drawings: not such a galling problem anymore. Talanta. 2010;81:412-7.

15. Oriola M, Campo G, Ruiz-Recasens C, Pedragosa N, Strlič M. Conservation management scenario appraisal for painting canvases at Museu Nacional d'Art de Catalunya. Stud Conserv. 2015;60:S193-9.
16. Zou X, Uesaka T, Gurnagul N. Prediction of paper permanence by accelerated aging. Part I: kinetic analysis of the aging process. Cellulose. 1996:3:243-67.

17. Erhardt D, Tumosa CS, Mecklenburg MF. Material consequences of the aging of paper, 12th Preprints of the Triennial ICOM-CC meeting, vol. 2. London: James \& James; 1999. p. 501-6.

18. SurveNIR website, http://www.science4heritage.org/survenir/collection. Accessed 8th July 2015.

19. Trafela T, Strlič M, Kolar J, Lichtblau DA, Anders M, Mencigar DP, Pihlar B. Nondestructive analysis and dating of historical paper based on IR spectroscopy and chemometric data evaluation. Anal Chem. 2007;79:6319-23.

20. Kolar J, Malešič J, Kočar D, Strlič M, De Bruin G, Koleša D. Characterisation of paper containing iron gall ink using size exclusion chromatography. Polym Degrad Stab. 2012;97:2212-6.

21. Dillon C, Lindsay W, Taylor J, Fouseki K, Bell N, Strlič M. Collections demography: stakeholders' views on the lifetime of collections. In: Ashley-Smith J, Burmester A, Eibl M, editors. Climate for collections conference, Munich, Doerner Institut, 7-9 November 2012, Postprints. London: Archetype; 2013. p. 45-58.

\section{Publish with ChemistryCentral and every scientist can read your work free of charge \\ "Open access provides opportunities to our colleagues in other parts of the globe, by allowing anyone to view the content free of charge."}

W. Jeffery Hurst, The Hershey Company.

- available free of charge to the entire scientific community

- peer reviewed and published immediately upon acceptance

- cited in PubMed and archived on PubMed Central

- yours - you keep the copyright

Submit your manuscript here:

http://www.chemistrycentral.com/manuscript/ 